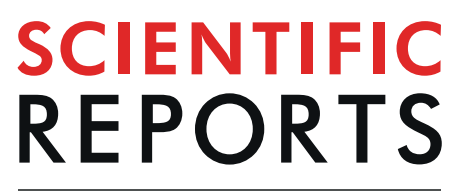

natureresearch

\title{
SP70-Targeted Imaging for the Early Detection of Lung Adenocarcinoma
}

\author{
Jian Xu ${ }^{1,2}$, Shichang Zhang ${ }^{1,2}$, Wei Zhang ${ }^{1}$, Erfu Xie ${ }^{1}$, Min Gu ${ }^{1}$, Yue Wang ${ }^{1}$, Lu Yang ${ }^{1}$, \\ Bingfeng Zhang ${ }^{1}$, Jiexin Zhang ${ }^{1}$, Chunrong Gu ${ }^{1}$, Ting $\mathrm{Xu}^{1}$, Daqian $\mathrm{Li}^{1}$, Fang Wang ${ }^{1}$, \\ Peijun Huang ${ }^{1}$ \& Shiyang Pan $^{1 *}$
}

NJ001 is a monoclonal antibody that can specifically recognize the SP70 antigen on lung adenocarcinoma cells. The goal of this study was to explore its utility in targeted imaging. Subcutaneous xenograft and orthotopic lung tumor implantation BALB/c mouse models were established. Near-infrared fluorescent CF750-labeled NJ001 was injected into two tumor mouse models. Mice that received orthotopic lung tumor implantation were also injected with NJ001-conjugated nanomagnetic beads intravenously, and then underwent micro-CT scanning. Meanwhile, mice with lung tumor were intravenously injected with normal saline and bare nanomagnetic beads as a control. Fluorescence could be monitored in the mice detected by anti-SP70 fluorescence imaging, which was consistent with tumor burden. Signal intensities detected with SP70-targeted micro-CT scans were greater than those in control mice. More importantly, orthotopic tumor lesions could be found on the fourth week with SP70-targeted imaging, which was 2 weeks earlier than detection in the control. Our results suggest that SP70 is a promising target for molecular imaging, and molecularly targeted imaging with an NJ001-labeled probe could be applied for the early detection of lung adenocarcinoma.

Lung cancer is the leading cause of cancer-related death in humans ${ }^{1,2}$. Approximately $1,590,000$ people died from lung cancer in $2012^{3}$. Five-year survival rates vary from $4-17 \%$, depending on stage and regional differences ${ }^{4}$. Late diagnosis makes treatment options challenging, but early detection could significantly reduce mortality in lung cancer patients ${ }^{5-7}$.

Different imaging modalities can be used for lung cancer detection, such as computed tomography (CT), magnetic resonance imaging (MRI), and ${ }^{18} \mathrm{~F}$-fluorodeoxyglucose positron emission tomography and computed tomography (FDG-PET/CT). However, they lack sufficient specificity for the early detection of tumors ${ }^{8}$. Molecularly targeted tumor imaging with a specific monoclonal antibody is superior to conventional nonspecific imaging technologies ${ }^{9,10}$. Nevertheless, there are few reports on molecularly targeted imaging for the detection of lung cancer, because of the lack of molecular targets with high specificity for lung cancer.

SP70 protein is a novel tumor marker of non-small-cell lung cancer (NSCLC), especially lung adenocarcinoma ${ }^{11}$. SP70's monoclonal antibody NJ001 could specifically recognize and react to lung adenocarcinoma cells ${ }^{12}$. In this study, we aimed to demonstrate the feasibility of SP70-targeted imaging with NJ001-conjugated nanomagnetic beads (immuno-nanomagnetic beads) in lung adenocarcinoma and the potential for its use in real-time monitoring and early detection.

\section{Results}

Bioluminescence intensity correlated with cell number. Both SPC-A1-luc and U87-luc cells were diluted by a serial 2 -fold dilution method, and then luciferase activity was measured. Bioluminescence intensity was highly correlated with the total number of cells $\left(R^{2}>0.9900\right)$ for both cell lines (Fig. S1). Furthermore, the average luciferase activity value of SPC-A1-luc in vitro was 1,223 photons/sec/cell.

SP70-targeted fluorescence imaging in subcutaneous xenograft mouse models. DIF showed that SP70 was located on the SPC-A1-luc cell membrane and in the cytoplasm but was not expressed in U87-luc cells (Fig. 1A). After subcutaneous implantation, three mice bearing subcutaneous SPC-A1-luc cell tumors were 
A
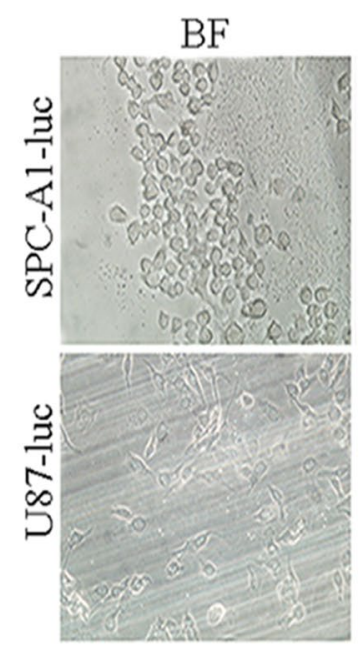
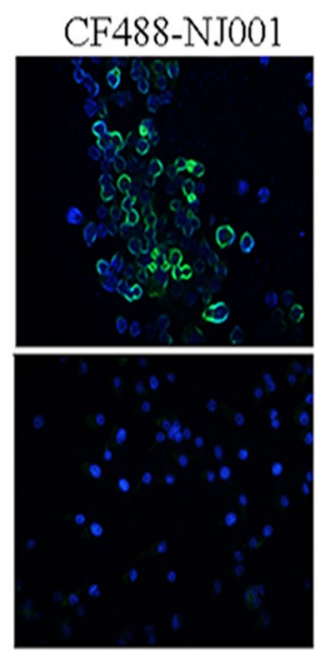

B

Week 3

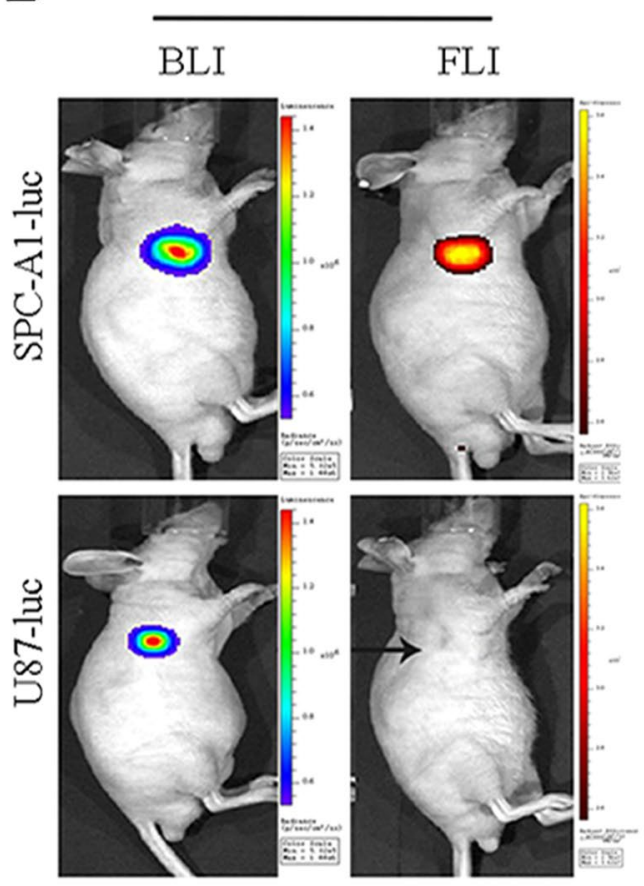

Figure 1. SP70-targeted fluorescence imaging in subcutaneous xenograft mouse models. (A) SP70 was located on the SPC-A1-luc cell membrane and in the cytoplasm, but was not expressed in U87-luc cells. (B) SPC-A1luc subcutaneous xenograft tumor could be detected by fluorescence imaging using NIR fluorescence CF750 (red)-labeled NJ001 3 weeks after inoculation. In contrast, U87-luc xenograft tumors could not be detected by fluorescence imaging (black arrow).

monitored weekly by both BLI and SP70-targeted fluorescence imaging in parallel. Three mice injected with U87-luc cells were used as controls. For SP70-targeted fluorescence imaging, fluorescent signals could be detected in the subcutaneous SPC-A1-luc mice starting in the third week. However, there were no fluorescent signals in the mice bearing glioma U87-luc cells (Fig. 1B). BLI could detect SPC-A1-luc xenograft tumor development by day 7 , and bioluminescence intensity increased in parallel with tumor volume measured by calipers.

SP70-targeted fluorescence imaging in orthotopic lung tumor models. Three mice with SPC-A1-luc orthotopic xenograft tumors were imaged with both BLI and fluorescence imaging. As shown in Fig. 2, SP70-targeted fluorescence imaging could detect the lesions at the third week. Meanwhile, the total lung BLI photon count was $7.2 \times 10^{5}$ photons/sec. Since in vitro BLI analysis showed that the luciferase activity value of a single SPC-A1-luc cell was 1,223 photons/sec, it was inferred that the tumor cell number reached approximately 600 on the third week.

Immuno-nanomagnetic bead characterization. NJ001 antibody-coated and noncoated nanomagnetic beads were characterized using transmission electron micrograph (TEM) with phosphotungstic acid staining (Fig. 3).

Signal enhancement of SP70-targeted micro-CT scan in orthotopic lung tumor models. After injection of the immuno-nanomagnetic beads, the micro-CT signal intensity of the orthotopic lung tumors increased significantly. The image density peaked at $4 \mathrm{~h}$ after injection of the immuno-nanomagnetic beads. The tumor grayscale index in the immuno-nanomagnetic beads group was the highest among the three groups (Fig. 4).

Tumor lesions could be detected at the sixth week in the mice injected with normal saline or bare nanomagnetic beads but were visible by the fourth week in the mice receiving immuno-nanomagnetic beads (Fig. 5). In other words, orthotopic tumor lesions could be found 2 weeks earlier by SP70-targeted micro-CT scan compared with routine micro-CT scan.

\section{Discussion}

The insidious onset of lung adenocarcinoma usually results in metastases at the time of initial diagnosis ${ }^{13,14}$. Early diagnosis of lung adenocarcinoma is challenging. Traditional imaging examinations such as CT and MRI are based on changes in tissue morphology $y^{15-18}$, and often lack sufficient specificity to fully predict a tumor's behavior $^{19-21}$. Therefore, it is essential to find tumor-specific molecules and establish reliable imaging methods for accurate diagnosis. 
A

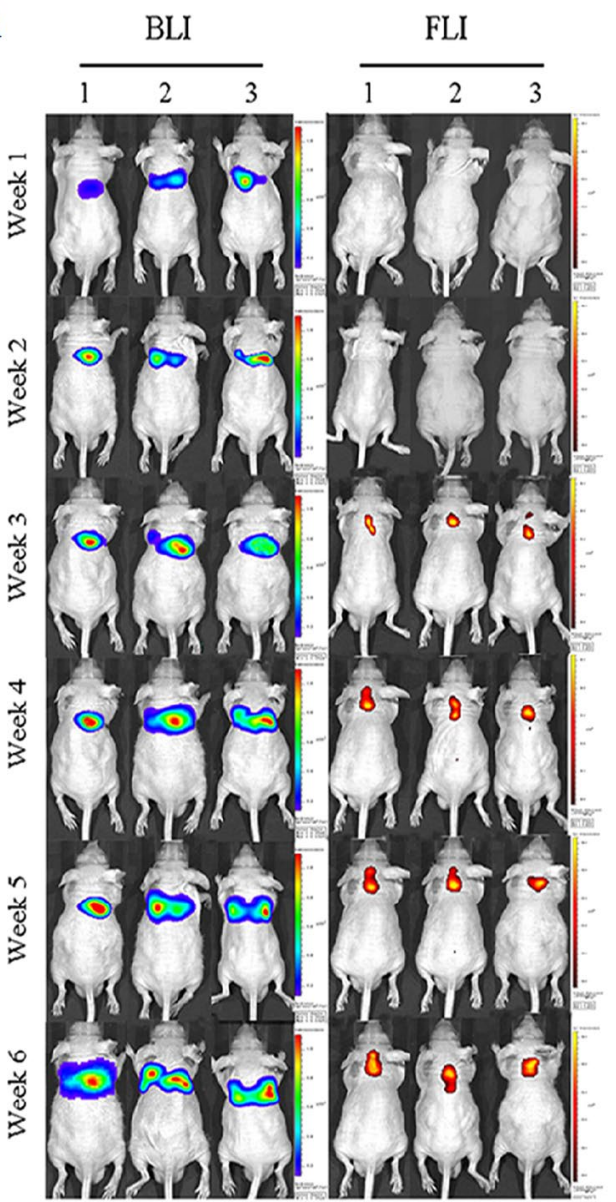

B

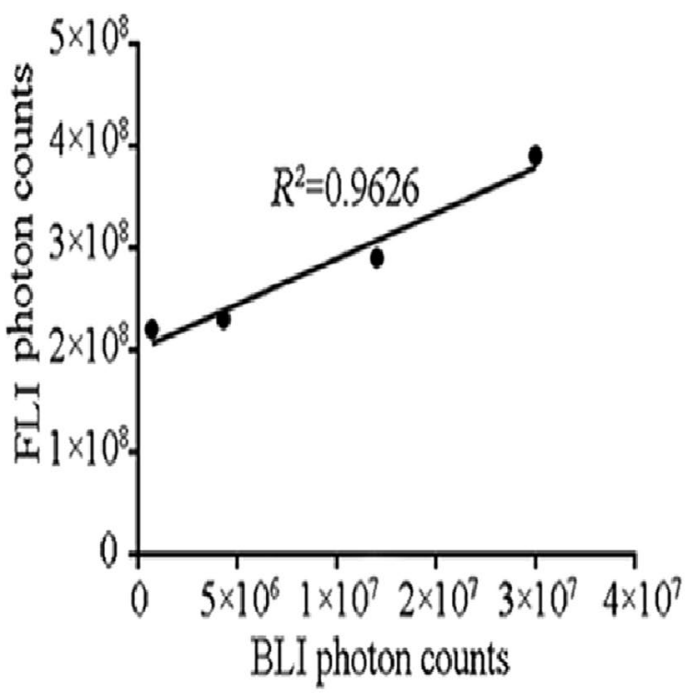

Figure 2. Tumor monitoring with SP70-targeted fluorescence imaging in orthotopic lung tumor implantation models. (A) Three mice with SPC-A1-luc orthotopic xenograft tumors were imaged by both BLI (left) and fluorescence imaging (right) at the $3^{\text {rd }}, 6^{\text {th }}$ and $9^{\text {th }}$ weeks. (B) FLI photon counts were correlated with BLI photon counts.
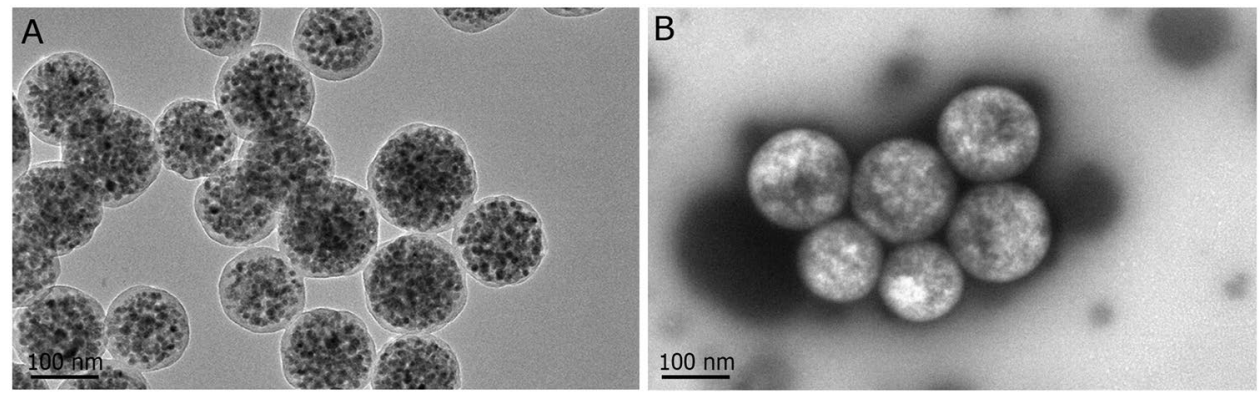

Figure 3. Immuno-nanomagnetic beads characterization with TEM. (A) TEM image of the nanomagnetic beads, showing a diameter of approximately $180 \mathrm{~nm}$; (B) TEM image of NJ001-conjugated nanomagnetic beads, using phosphotungstic acid for background staining.

The SP70 protein is expressed in lung adenocarcinoma but not in normal tissue, blood cells or other cell lines, such as U87 (a glioma cell line). And NJ001 also exhibits anti-tumor activity against NSCLC both in vitro and in vivo ${ }^{12}$. SP70 is detectable in the serum of patients with lung adenocarcinoma. We also found that SP70 is a key protein that can regulate the expression of numerous genes (GEO accession number: GSE59655), promoting cancer cell proliferation and metastasis. Molecularly targeted imaging will allow clinicians to not only to see where a microscopic tumor is located in the body at an early stage, but also to specifically visualize the biological characteristics of tumors ${ }^{22-24}$. However, biomarker discovery becomes challenging because only a few biomarkers can be both diagnostic and prognostic ${ }^{25}$. Thyroid transcription factor-1 (TTF-1) and napsin A have been well known as 


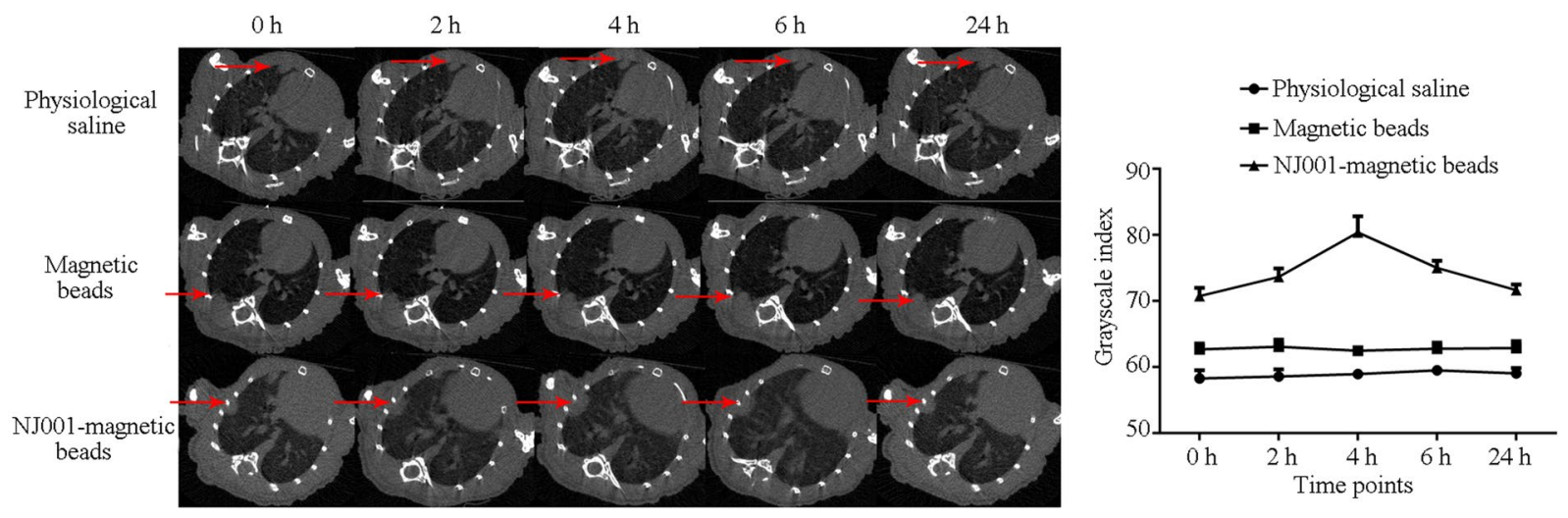

Figure 4. Signal enhancement in SP70-targeted micro-CT scan in orthotopic lung tumor implantation models. Micro-CT scan at 0, 2, 4, 6 and $24 \mathrm{~h}$ after NJ001 conjugated nanomagnetic beads or control injection in the sixth week. The image density increased and peaked at $4 \mathrm{~h}$ postinjection with NJ001-conjugated nanomagnetic beads.

\section{CT}

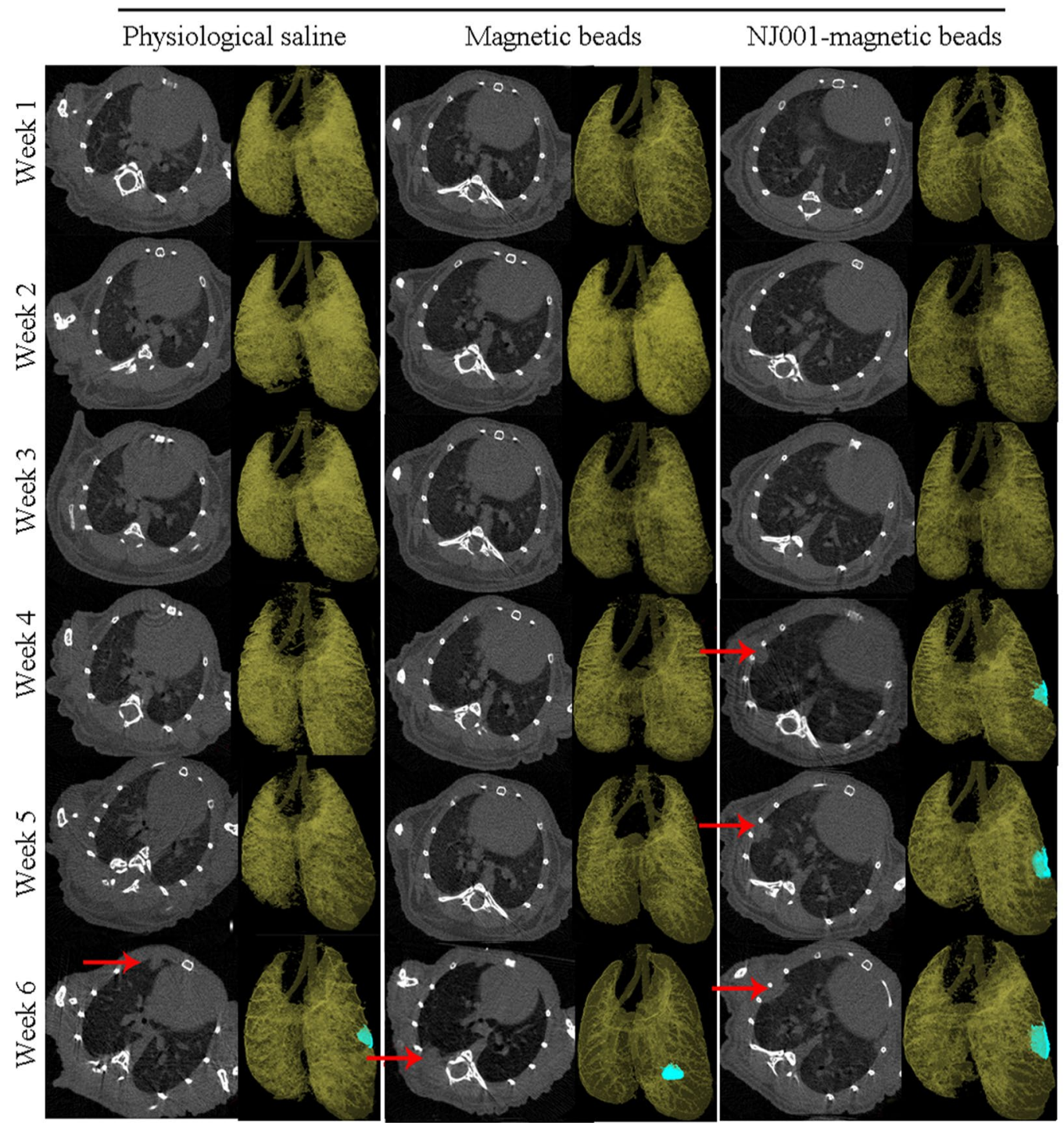

Figure 5. Earlier tumor detection with SP70-targeted micro-CT imaging. SPC-A1-luc orthotopic xenograft tumors were detected by micro-CT scan weekly. Each group contained three mice. One image of each group is shown. 
lung adenocarcinoma biomarkers for immunohistochemistry ${ }^{26}$. However, they are not suitable for the application of targeted imaging technology due to their nononcogenic origins. Recently, Predina et al. reported that folate receptor alpha $(\mathrm{FR} \alpha)$-targeted intraoperative molecular imaging might be used during surgery ${ }^{27}$.

Three kinds of in vivo molecular imaging techniques were developed in this study: BLI based on the activity of luciferase that catalyzes the substrate luciferin in transfected cells, targeted fluorescence imaging with CF750-NJ001, and targeted micro-CT with NJ001-conjugated nanomagnetic beads. Since bioluminescence signal intensity positively correlates with tumor volume, we could perform a quantitative assessment of tumor growth in vivo. It was reported that molecular fluorescence imaging with near-infrared fluorescence could be used for detecting tumors and guiding surgery ${ }^{28-30}$. We chose the near infrared fluorescent dye CF750 to label NJ001 due to the wavelength's enhanced tissue penetrability and the dye's favorable pharmacokinetic properties. The fluorescent signal perfectly matched the bioluminescence signal. Conversely, there were no fluorescent signals in mice bearing glioma U87-luc cells, which were negative for the SP70 antigen. These results demonstrated that CF750-NJ001 had high specificity for lung subcutaneous xenografts.

Furthermore, lesions in orthotopic lung models could be detected by SP70-targeted fluorescence imaging at the third week, while positive signals were found at the sixth week by conventional micro-CT. Considering photon attenuation when penetrating the body cavity, lesions may contain more than 600 tumor cells. Routine CT scans can only detect lesions of more than $1.5 \mathrm{~mm}$ lesions which contain approximately $10^{6}$ tumor cells. This result indicates that SP70-targeted fluorescence imaging is much more sensitive than traditional imaging techniques. Therefore, SP70 acts as an oncogenic protein and could be a target of molecular imaging, even for micrometastasis. Because of the weak penetration ability in the human body, SP70-targeted fluorescence imaging might only be used to locate lung adenocarcinomas and identify positive margins during surgery.

$\mathrm{CT}$ is the first-line imaging tool for lung cancer diagnosis. Though contrast agents can enhance the resolution of CT, they cannot improve its ability to differentiate malignant from benign tumors. Current contrast agents are mainly (approximately 90\%) excreted by kidneys. A larger dose of contrast agent often leads to the occurrence of nephropathy ${ }^{31}$. Nanotechnology could be used in molecularly targeted imaging. Nanoparticles easily penetrate cancerous tissue capillaries ${ }^{32}$, and can be deposited preferentially in cancer tissue. Recently, Patrick et al. used CNA35-conjugated gold nanoparticles as a CT contrast agent for the molecular imaging of myocardial scars ${ }^{33}$. In our study, the diameter of the nanomagnetic beads was $180 \mathrm{~nm}$, so they could easily be distributed into cancer tissue. NJ001-conjugated nanomagnetic beads could specifically identified lung adenocarcinoma cells, and molecularly targeted enhancement significantly improved the sensitivity of micro-CT imaging for monitoring tumor growth. Lesions were detected at the fourth week in the SP70-targeted micro-CT group, 2 weeks (one third of the amount of time) earlier than in the groups injected with normal saline and bare nanomagnetic beads controls. It could be inferred that SP70-targeted imaging could greatly shorten the period from tumor onset to diagnosis, and therefore accomplish the aim of detecting the early stage of lung adenocarcinoma. Although the antigenicity of the murine monoclonal antibody NJ001 was minimized with conjugation to the nanomagnetic beads, its biosafety and metabolic properties remain to be determined.

In conclusion, SP70-targeted imaging can markedly improve the detection of lung adenocarcinoma detection. Molecularly targeted imaging with NJ001-labeled probes may have precision medical applications for the early diagnosis of lung adenocarcinoma.

\section{Materials and Methods}

Materials. Superparamagnetic polymer nanospheres were purchased from Shanghai Allrun Nano New Science \& Technology Ltd. (Shanghai, China). Bovine serum albumin (BSA), ethylcarbodiimide (EDC) and N-hydroxysuccinimide (NHS) were purchased from Sigma-Aldrich (St. Louis, MO, USA). The CF488 Dye Antibody Labeling Kit and the CF750 Dye Antibody Labeling Kit, which are both fluorescent succinyl ester dye kits, were purchased from Biotium (Hayward, CA, USA). D-luciferin was purchased from Fanbo Biochemicals (Beijing, China).

Cell culture. SPC-A1-luc cells (SP70-positive) and U87-luc cells (SP70-negative), cell lines derived from human lung adenocarcinoma and human glioma, respectively, that express luciferase by stable transfection with the firefly luciferase gene, were purchased (Shanghai Baidaian Company, Shanghai, China). Both cell lines were cultured in Roswell Park Memorial Institute (RPMI) 1640 medium containing $50 \mathrm{U} / \mathrm{mL}$ penicillin, $50 \mathrm{U} / \mathrm{mL}$ streptomycin, and 10\% fetal bovine serum (Invitrogen, Carlsbad CA, USA). Cells were grown in an incubator with $5 \% \mathrm{CO}_{2}$ at $37^{\circ} \mathrm{C}$.

Animal models. Four-week-old male BALB/c nude mice (Shanghai SLAC Laboratory Animal Co. Ltd. Shanghai, China) were maintained in a pathogen-free environment. All procedures were conducted in accordance with the Animal Care and Use Committee guidelines of Nanjing Medical University. To establish subcutaneous xenograft mouse models, SPC-A1-luc and control U87-luc cells were harvested in the logarithmic growth phase, counted, and resuspended in phosphate-buffered saline (PBS) to a final density of $5 \times 10^{7} \mathrm{cells} / \mathrm{mL}$. Next, $5 \times 10^{6} \mathrm{SPC}-\mathrm{A} 1$-luc cells or U87-luc cells suspended in $0.1 \mathrm{~mL}$ of sterile PBS were implanted subcutaneously into the right flank of each mouse. For an orthotopic lung tumor implantation mouse model, $5 \times 10^{6} \mathrm{SPC}$-A1-luc cells suspended in $0.1 \mathrm{~mL}$ of sterile PBS were injected into mice intravenously via the tail vein. All animal studies were performed with the approval of the Animal Care and Use Committee of Nanjing Medical University.

NJ001-conjugated nanomagnetic beads (immuno-nanomagnetic beads). Superparamagnetic polymer nanospheres with diameters of $180 \mathrm{~nm}$ were conjugated with NJ001 antibody. First, 2 mg nanomagnetic beads were activated with $200 \mu \mathrm{L}$ of $5 \mathrm{mg} / \mathrm{mL}$ EDC and NHS in a $37^{\circ} \mathrm{C}$ water bath for $30 \mathrm{~min}$, followed by three washes with phosphate-buffered saline with tween 20 (PBST). Second, the beads were mixed with $50 \mu \mathrm{L}$ of $5 \mathrm{mg} / \mathrm{mL}$ 
NJ001 antibody and again incubated in a $37^{\circ} \mathrm{C}$ water bath for $4 \mathrm{~h}$. Third, after three more washes with PBST, the beads were resuspended in $1 \mathrm{~mL}$ of PBST containing 5\% BSA and gently shaken at $4{ }^{\circ} \mathrm{C}$ overnight. Finally, the supernatant was removed, and the beads were resuspended in $200 \mu \mathrm{L}$ of PBST and kept at $4{ }^{\circ} \mathrm{C}$.

Immuno-nanomagnetic bead characterization. TEM were taken by using a JEM-2100 HR transmission electron microscope (JEOL, Tokyo, Japan). The nanomagnetic bead sample $(5 \mu \mathrm{L})$ was dropped onto copper 200-mesh grids that were pretreated with UV light to reduce static electricity. After $30 \mathrm{~min}$, the solvent was drained with filter paper, and a phosphotungstic acid stain solution ( $1 \%$ by weight, adjusted to $\mathrm{pH} 6.0)$ was applied for $30 \mathrm{~s}$. After drying, TEM imaging was performed.

Direct immunofluorescence (DIF) analysis. The Dye Antibody Labeling Kit was used to develop the fluorescently dyed probe CF488-NJ001 for in vitro DIF. SPC-A1-luc and U87-luc cells were grown on coverslips, fixed in $95 \%$ ethanol for $15 \mathrm{~min}$ at room temperature and blocked with $5 \% \mathrm{BSA}$ for $30 \mathrm{~min}$ at $37^{\circ} \mathrm{C}$. After washing three times with PBS, the coverslips were incubated with CF488-NJ001 (1:200 dilution) for $30 \mathrm{~min}$ at $37^{\circ} \mathrm{C}$. After three washes, a fluorescent dye, 4',6-diamidino-2-phenylindole (DAPI) was subsequently added for nuclear staining. Samples were examined using an Olympus IX71 inverted fluorescence microscope (Olympus Optical Co. Ltd, Tokyo, Japan) coupled with a charge-coupled device (CCD) camera (ProgRes, Jenoptik/Jena, Germany).

Bioluminescence imaging (BLI). Mice were injected intraperitoneally with $150 \mathrm{mg} / \mathrm{kg}$ of D-luciferin in PBS, and imaged with IVIS 2000 imaging system (Caliper Life Sciences, Hopkinton MA, USA). Analyses of the BLI images were performed using Living Image software from Caliper Life Sciences by drawing regions of interest over the tumor region and obtaining maximum values in photons per second per $\mathrm{cm}^{2}$ per steradian or total flux as photons per second. BLI was repeated weekly.

Fluorescence imaging (FLI). Fluorescence imaging was performed using the CF750 Dye Antibody Labeling Kit, a fluorescent succinyl ester dye kit, to label NJ001 for in vivo fluorescence imaging analysis. A dose of $5 \mu \mathrm{g}$ of CF750-NJ001 in $100 \mu \mathrm{L}$ PBS was injected via the tail vein one week after the cell inoculation and weekly thereafter. The fluorescence intensity in each mouse was assessed $48 \mathrm{~h}$ after injection using the same IVIS 2000 imaging system with an excitation wavelength of $755 \mathrm{~nm}$ and an emission wavelength of $800 \mathrm{~nm}$. Fluorescence from the ROI was defined manually, and fluorescence efficiency was expressed as (photons/s) $\div\left(\mu \mathrm{W} / \mathrm{cm}^{2}\right)^{34}$. Images were analyzed using Living Image software.

Micro-computed tomography (micro-CT). Orthotopic mouse models were imaged with BLI to monitor tumor growth one week after SPC-A1-luc cell inoculation. Nine mice with no significant difference in tumor size were selected for the following micro-CT scans. Three orthotopic mice were injected intravenously with $100 \mu \mathrm{L}$ NJ001-conjugated nanomagnetic beads, three mice were injected with $100 \mu \mathrm{L}$ normal saline and three mice were injected with bare nanomagnetic beads as controls. Micro-CT scans were acquired at 0,2, 4, 6 and $24 \mathrm{~h}$ after injection to determine tumor lesion and size. Micro-CT was performed on a SkyScan 1176 (SkyScan NV, Kontich, Belgium), a small animal imager, at $50 \mathrm{kV}$ and $490 \mu \mathrm{A}$. A total of $360^{\circ}$ views were acquired at $1^{\circ}$ angle increments, each for an exposure time of $120 \mathrm{~ms}$, to give a resolution of $35 \mu \mathrm{m}$ using external respiratory gating. SkyScan software was used for multiplanar and $3 \mathrm{D}$ image reconstruction.

Statistical analysis. Statistical analysis was conducted using SPSS Statistics v20.0 (SPSS Inc., Chicago IL, USA) and GraphPad Prism v6.0 (GraphPad Software, San Diego CA, USA). Dunnett's test was used to compare the experimental group to the control groups undergoing micro-CT scans. The correlation was analyzed by the Pearson correlation coefficient. All statistical assessments were performed in a two-sided setting, using a significance cut-off of 0.05 .

\section{Data availability}

All data generated or analysed during this study are included in this published article (and its Supplementary Information Files).

Received: 13 February 2019; Accepted: 27 January 2020;

Published online: 13 February 2020

\section{References}

1. Siegel, R. L., Miller, K. D. \& Jemal, A. Cancer Statistics, 2017. CA: a cancer journal for clinicians 67, 7-30, https://doi.org/10.3322/ caac.21387 (2017).

2. Cheng, T. D. et al. The International Epidemiology of Lung Cancer: Latest Trends, Disparities, and Tumor Characteristics. Journal of thoracic oncology: official publication of the International Association for the Study of Lung Cancer, https://doi.org/10.1016/j. jtho.2016.05.021 (2016)

3. Islami, F., Torre, L. A. \& Jemal, A. Global trends of lung cancer mortality and smoking prevalence. Translational lung cancer research 4, 327-338, https://doi.org/10.3978/j.issn.2218-6751.2015.08.04 (2015).

4. Hirsch, F. R. et al. Lung cancer: current therapies and new targeted treatments. Lancet (London, England) 389, 299-311, https://doi. org/10.1016/s0140-6736(16)30958-8 (2017).

5. Bade, M. et al. Effect of smoking cessation counseling within a randomised study on early detection of lung cancer in Germany. Journal of cancer research and clinical oncology 142, 959-968, https://doi.org/10.1007/s00432-015-2105-0 (2016).

6. Lin, G., Li, Y., Chen, S. \& Jiang, H. Integrated Chinese-western therapy versus western therapy alone on survival rate in patients with non-small-cell lung cancer at middle-late stage. Journal of traditional Chinese medicine =Chung $i$ tsa chih ying wen pan 33, 433-438 (2013).

7. Zukotynski, K. A. \& Gerbaudo, V. H. Molecular Imaging and Precision Medicine in Lung Cancer. PET clinics 12, 53-62, https://doi. org/10.1016/j.cpet.2016.08.008 (2017) 
8. Youngstein, T. et al. FDG Uptake by Prosthetic Arterial Grafts in Large Vessel Vasculitis Is Not Specific for Active Disease. JACC. Cardiovascular imaging 10, 1042-1052, https://doi.org/10.1016/j.jcmg.2016.09.027 (2017).

9. Zhang, X., Soori, G., Dobleman, T. J. \& Xiao, G. G. The application of monoclonal antibodies in cancer diagnosis. Expert review of molecular diagnostics 14, 97-106, https://doi.org/10.1586/14737159.2014.866039 (2014).

10. Freise, A. C. \& Wu, A. M. In vivo imaging with antibodies and engineered fragments. Molecular immunology 67, 142-152, https:// doi.org/10.1016/j.molimm.2015.04.001 (2015).

11. Liu, J. et al. Serum SP70 is a sensitive predictor of chemotherapy response in patients with advanced nonsmall cell lung cancer. Cancer medicine, https://doi.org/10.1002/cam4.1555 (2018).

12. Pan, S. et al. The study on newly developed McAb NJ001 specific to non-small cell lung cancer and its biological characteristics. PloS one 7, e33009, https://doi.org/10.1371/journal.pone.0033009 (2012).

13. Lim, S. M. et al. Genomic profiling of lung adenocarcinoma patients reveals therapeutic targets and confers clinical benefit when standard molecular testing is negative. Oncotarget 7, 24172-24178, https://doi.org/10.18632/oncotarget.8138 (2016).

14. Eguchi, T. et al. Cell cycle progression score is a marker for five-year lung cancer-specific mortality risk in patients with resected stage I lung adenocarcinoma. Oncotarget 7, 35241-35256, https://doi.org/10.18632/oncotarget.9129 (2016).

15. Wienbeck, S., Lotz, J. \& Fischer, U. Review of clinical studies and first clinical experiences with a commercially available cone-beam breast CT in Europe. Clinical imaging 42, 50-59, https://doi.org/10.1016/j.clinimag.2016.11.011 (2017).

16. Heye, T. et al. Detection and size of pulmonary lesions: how accurate is MRI? A prospective comparison of CT and MRI. Acta radiologica (Stockholm, Sweden: 1987) 53, 153-160, https://doi.org/10.1258/ar.2011.110445 (2012).

17. Cai, X. et al. Enabling Prussian Blue with Tunable Localized Surface Plasmon Resonances: Simultaneously Enhanced Dual-Mode Imaging and Tumor Photothermal Therapy. 10, 11115-11126, https://doi.org/10.1021/acsnano.6b05990 (2016).

18. Stillwater, L., Koenig, J., Maycher, B. \& Davidson, M. 3D-MR vs. 3D-CT of the shoulder in patients with glenohumeral instability. Skeletal radiology 46, 325-331, https://doi.org/10.1007/s00256-016-2559-4 (2017).

19. Nakajima, T. \& Yasufuku, K. Early lung cancer: methods for detection. Clinics in chest medicine 34, 373-383, https://doi. org/10.1016/j.ccm.2013.04.005 (2013).

20. Jett, J. R. Limitations of screening for lung cancer with low-dose spiral computed tomography. Clinical cancer research: an official journal of the American Association for Cancer Research 11, 4988s-4992s, https://doi.org/10.1158/1078-0432.ccr-05-9000 (2005).

21. Olafsen, T. \& Wu, A. M. Antibody vectors for imaging. Seminars in nuclear medicine 40, 167-181, https://doi.org/10.1053/j. semnuclmed.2009.12.005 (2010).

22. Jimenez-Bonilla, J. F. et al. The Role of PET/CT Molecular Imaging in the Diagnosis of Recurrence and Surveillance of Patients Treated for Non-Small Cell Lung Cancer. Diagnostics (Basel, Switzerland) 6, https://doi.org/10.3390/diagnostics6040036 (2016).

23. Trousil, S. et al. Positron emission tomography imaging with 18F-labeled ZHER2:2891 affibody for detection of HER2 expression and pharmacodynamic response to HER2-modulating therapies. Clinical cancer research: an official journal of the American Association for Cancer Research 20, 1632-1643, https://doi.org/10.1158/1078-0432.ccr-13-2421 (2014).

24. Yoo, M. K. et al. Folate-PEG-superparamagnetic iron oxide nanoparticles for lung cancer imaging. Acta biomaterialia 8, 3005-3013, https://doi.org/10.1016/j.actbio.2012.04.029 (2012).

25. O'Neill, A. F. et al. Targeted imaging of Ewing sarcoma in preclinical models using a 64Cu-labeled anti-CD99 antibody. Clinical cancer research: an official journal of the American Association for Cancer Research 20, 678-687, https://doi.org/10.1158/1078-0432. ccr-13-1660 (2014).

26. Veronesi, G., Bianchi, F., Infante, M. \& Alloisio, M. The challenge of small lung nodules identified in CT screening: can biomarkers assist diagnosis? Biomarkers in medicine 10, 137-143, https://doi.org/10.2217/bmm.15.122 (2016).

27. Predina, J. D., Okusanya, O. A., D. N., Low, P. \& Singhal, S. Standardization and Optimization of Intraoperative Molecular Imaging for Identifying Primary Pulmonary Adenocarcinomas. Molecular imaging and biology: MIB: the official publication of the Academy of Molecular Imaging 20, 131-138, https://doi.org/10.1007/s11307-017-1076-8 (2018).

28. Hernandez, R. et al. CD146-targeted immunoPET and NIRF Imaging of Hepatocellular Carcinoma with a Dual-Labeled Monoclonal Antibody. Theranostics 6, 1918-1933, https://doi.org/10.7150/thno.15568 (2016).

29. Hoogstins, C. E. et al. A Novel Tumor-Specific Agent for Intraoperative Near-Infrared Fluorescence Imaging: A Translational Study in Healthy Volunteers and Patients with Ovarian Cancer. Clinical cancer research: an official journal of the American Association for Cancer Research 22, 2929-2938, https://doi.org/10.1158/1078-0432.ccr-15-2640 (2016).

30. Sonn, G. A. et al. Fluorescent Image-Guided Surgery with an Anti-Prostate Stem Cell Antigen (PSCA) Diabody Enables Targeted Resection of Mouse Prostate Cancer Xenografts in Real Time. Clinical cancer research: an official journal of the American Association for Cancer Research 22, 1403-1412, https://doi.org/10.1158/1078-0432.ccr-15-0503 (2016).

31. Yin, W. J. et al. Preprocedural Prediction Model for Contrast-Induced Nephropathy Patients. Journal of the American Heart Association 6, https://doi.org/10.1161/jaha.116.004498 (2017).

32. Wang, X., Yang, L., Chen, Z. G. \& Shin, D. M. Application of nanotechnology in cancer therapy and imaging. CA: a cancer journal for clinicians 58, 97-110, https://doi.org/10.3322/ca.2007.0003 (2008).

33. Kee, P. H. \& Danila, D. CT imaging of myocardial scar burden with CNA35-conjugated gold nanoparticles. Nanomedicine: nanotechnology, biology, and medicine 14, 1941-1947, https://doi.org/10.1016/j.nano.2018.06.003 (2018).

34. Gao, J. et al. In vivo tumor-targeted fluorescence imaging using near-infrared non-cadmium quantum dots. Bioconjugate chemistry 21, 604-609, https://doi.org/10.1021/bc900323v (2010).

\section{Acknowledgements}

This work was supported by grants from the National Natural Science Foundation of China (Grant ID 81672100), and the Key Laboratory for Laboratory Medicine of Jiangsu Province of China (Grant ID ZDXKB2016005) and was funded by the Priority Academic Program Development of Jiangsu Higher Education Institutions. The authors are grateful to the Animal Core Facility of Nanjing Medical University.

\section{Author contributions}

S.P. conceived the study and wrote the manuscript. J.X., S.Z. W.Z., E.X., M.G., Y.W., L.Y., B.Z. and D.L. performed the experiments. J.Z. performed the statistical analysis. C.G., T.X. and F.W. contributed methodological support. P.H. critically revised the manuscript.

\section{Competing interests}

The authors declare no competing interests.

\section{Additional information}

Supplementary information is available for this paper at https://doi.org/10.1038/s41598-020-59439-9.

Correspondence and requests for materials should be addressed to S.P. 
Reprints and permissions information is available at www.nature.com/reprints.

Publisher's note Springer Nature remains neutral with regard to jurisdictional claims in published maps and institutional affiliations.

(c) (i) Open Access This article is licensed under a Creative Commons Attribution 4.0 International License, which permits use, sharing, adaptation, distribution and reproduction in any medium or format, as long as you give appropriate credit to the original author(s) and the source, provide a link to the Creative Commons license, and indicate if changes were made. The images or other third party material in this article are included in the article's Creative Commons license, unless indicated otherwise in a credit line to the material. If material is not included in the article's Creative Commons license and your intended use is not permitted by statutory regulation or exceeds the permitted use, you will need to obtain permission directly from the copyright holder. To view a copy of this license, visit http://creativecommons.org/licenses/by/4.0/.

(C) The Author(s) 2020 